\title{
Syphilis sero-positivity among pregnant women attending public antenatal clinics: A five-year analysis from 15 public clinics in Gaborone, Botswana
}

\author{
AB Ganiyu ${ }^{a *}$, L Mason $^{b}$ and LH Mabuzac \\ ${ }^{a}$ Faculty of Medicine, Department of Family Medicine, University of Botswana, Gaborone, Botswana \\ ${ }^{b}$ Department of Health and Life Sciences Programme, University of Liverpool/Laureate Online Education, Amsterdam, The Netherlands \\ 'Department of Family Medicine and Primary Health Care, Sefako Makgatho Health Sciences University, Pretoria, South Africa \\ *Corresponding author, email: waleganiyu@gmail.com
}

\begin{abstract}
Background: The prevalence of syphilis in pregnancy varies across the globe and among different age groups within the same country. In sub-Saharan Africa, syphilis prevalence among pregnant women has been found to range from 2.5 to $18 \%$ among antenatal clinic attendees, with the highest prevalence in the age group 35-49 years. Also, it is higher in the rural than urban clinics.

Objectives: To determine trends in syphilis prevalence using the Venereal Disease Research Laboratory (VDRL) and the Rapid Plasma Reagin (RPR) test among pregnant women attending the public antenatal clinics in Gaborone, Botswana (2004-2008).

Study design: Cross-sectional study using routinely collected antenatal data.

Results: The overall syphilis prevalence amongst pregnant women in Gaborone, Botswana decreased from $2.96 \%$ (95\% Cl, $2.55-$ $3.37)$ in 2004 to $1.15 \%(95 \% \mathrm{Cl}, 0.89-1.41)$ in $2008(p<0.001)$. The age specific prevalence per total number of reactive VDRL/RPR was highest amongst pregnant women aged 26 to 30 years $(p<0.001)$ and lowest for those aged 16 to 20 years $(p<0.025)$ during the period 2004-2008. However, there were variations in syphilis prevalence rates within and between the clinics.

Conclusion: Syphilis sero-positivity in pregnancy in Gaborone, Botswana has been declining for the last five years, but was more prevalent amongst pregnant women aged 26 to 30 years with the lowest prevalence among those aged 16 to 20 years during the period 2004 to 2008. This decline may be attributed to a number of factors and, in particular, the adoption of the syndromic approach for management of sexually transmitted infections in the country.
\end{abstract}

Keywords: antenatal clinics, pregnant women, syphilis, sero-positivity, sero-prevalence

\section{Introduction}

The Venereal Disease Research Laboratory (VDRL) and the Rapid Plasma Reagin (RPR) tests are non-treponemal serological screening tests for syphilis. Since they are not specific for syphilis infection, the Fluorescent Treponemal Antibody-Absorption (FTA-ABS) and the Treponema Pallidum Hemaglutination Assay (TPHA), both treponemal-specific tests, are used to confirm syphilis infection (following a positive screening test through VDRL or RPR). ${ }^{1-3}$ According to the Republic of Botswana national guideline, at the time of the study, screening for syphilis was mandatory in the first and third trimesters of pregnancy with non-treponemal tests such as VDRL or RPR (depending on the available reagent). The decision to adopt this guideline was based on the high prevalence of syphilis in the country, the recommendation by the World Health Organization (WHO) for poorly resourced countries (like Botswana), and cost implications of carrying out treponemal tests. ${ }^{4-6}$ A positive VDRL/RPR was considered a sero-reactive case, and testing was considered complete if performed at least once per index pregnancy.

The trend in syphilis prevalence among pregnant women has been found to vary across the globe and among different age groups within the same country. ${ }^{4}$ While a decline in syphilis prevalence rates has been noted across Western Europe and the United States of America (USA), increasing rates have been reported in most developing countries. ${ }^{4}$ The WHO (2001) estimated that the prevalence rates ranged from $2.5 \%$ in Burkina Faso, $6.7 \%$ in the Central African Republic, $8.4 \%$ in South Africa to $17.4 \%$ in Cameroon. ${ }^{4}$ This was supported by another study that reported prevalence rates of syphilis in pregnancy in developing countries ranging from $2.5-17.0 \% .^{7}$ An overview of 23 studies on antenatal syphilis prevalence from 14 sub-Saharan African countries showed that the weighted mean for syphilis seroprevalence was $8.3 \%$, the median was $8.75 \%$ and the range was $0.9-20.8 \% .^{8}$ In South Africa, prevalence rates of $1.04-8.4 \%$ have been reported for syphilis in pregnancy, with a declining trend from $4.9 \%$ in $2000,2.8 \%$ in 2001 to $1.8 \%$ in $2006.4,7,9$

In the Republic of Botswana, the Ministry of Health reported that $4.8 \%$ in $2002,5 \%$ in 2003 and $3 \%$ in 2005 of pregnant women were RPR positive during National HIV/AIDS sentinel serosurveillance. $^{10}$ A cross-sectional study of 703 antenatal care attendees at 13 health facilities in Gaborone, Botswana found that $4.6 \%$ (32) of the attendees had active syphilis, with syphilis prevalence rates of up to $13.0 \%$ in rural areas. ${ }^{11,12}$ Another study showed a decline in the prevalence rate of active syphilis (defined as a positive RPR confirmed by either TPHA or FTA-ABS) from 18\% in 1993 to $2 \%$ in 2002 among women attending family planning clinics in Botswana. ${ }^{10}$ Possible reasons for the decline were the implementation of syndromic approach in the management of sexually transmitted infections, improved access to health care, and patient education on less risky sexual behavior. ${ }^{10}$

The aim of the study was to determine syphilis sero-positivity among pregnant women attending public antenatal clinics, in a five-year period (2004-2008) in all the fifteen (15) public primary health care facilities providing antenatal care in Gaborone, Botswana. 


\section{Ethical considerations}

Ethical approval was obtained from the research and ethics committees of the University of Liverpool (as per the communication from the Director of Studies-MPH program) and the Ministry of Health, Botswana (Ref. No: PPME 13/18/1 PS IV $\{50\})$. Permission to obtain data was obtained from the local government authority, Gaborone City Council.

\section{Methods \\ Study design}

This was a cross-sectional study to investigate syphilis prevalence among pregnant women attending public antenatal clinics for 2004-2008.

\section{Study settings}

This study was conducted in the 15 clinics that provide routine antenatal services located in Gaborone, Botswana. They were $\mathrm{BH} 1, \mathrm{BH} 2, \mathrm{BH} 3$, Block 6, Block 8, Block 9, Bontleng, BTA, Extension 2, Extension 14, Extension 15, Sebele, Phase 2, ONC and G-west.

\section{Study population}

The total number of patients attending these clinics was 31221 over the specified time period.

\section{Data source \& data collection}

Data were collected from the 7th to 25th of September 2009, from the antenatal registers using a structured data collecting sheet. Four variables were recorded from the files during the specified time period (2004-2008):

(1) Total number of pregnant women registered for antenatal care per year;

(2) Number of women with reactive VDRL/RPR per year;

(3) Individual age of the women with reactive VDRL/RPR; and,

(4) Number of women with reactive VDRL/RPR per age group per year.

The women were stratified into age groups: 16-20 years, $21-25$ years, $26-30$ years, $31-35$ years and $\geq 35$ years. We followed the age groups as reflected in the antenatal registry in Gaborone, Botswana. ${ }^{13}$

\section{Reliability and validity}

Validity of the data set was enhanced by a standardised record keeping system in all 15 clinics. These records were readily available and regularly updated by the attending midwife during each antenatal visit. Data recorded from the antenatal registers of the 15 clinics were found to be similar to the data at the District Health Team central office, where the data from the clinics were collated and analysed on a monthly basis.

Using the same data collection sheet consistently throughout the data collection process ensured reliability. Data were coded using a clinic code in order to distinguish each study site. The reliability of data management was further enhanced by double data entry.

\section{Data analysis}

Overall trends in syphilis prevalence, prevalence by age group and prevalence by clinic were determined. The data were captured, edited and cleaned using SPSS v14.0 for Windows software, then exported to Microsoft Excel for statistical analysis. The Chi-square $\left(\chi^{2}\right)$ test was used and significance level for trend in syphilis prevalence by age group per year calculated. Statistical significance was set at a $p$-value of $\leq 0.05$.

\section{Results}

\section{Trends in syphilis prevalence}

Table 1 shows the number and proportion of registered pregnant women with reactive VDRL/RPR. This table demonstrates a gradual decline in the proportion of registered pregnant women with syphilis sero-positivity from $2.96 \%(95 \% \mathrm{Cl}, 2.55-3.37)$ to $1.15 \%(95 \% \mathrm{Cl}, 0.89-1.41)$ with a $p<0.001\left(\chi^{2}=74.85\right.$; degrees of freedom $=4$ ) over the five-year period, 2004-2008.

\section{Trends in syphilis prevalence per total number of reactive VDRL/RPR by age group for combined clinics per year, 2004-2008}

The sero-prevalence of syphilis in the total number of reactive VDRL/RPR by age group over the five-year period was the highest amongst pregnant women aged 26 to 30 years $(p<0.001)$, and lowest amongst women aged 16 to 20 years $(p<0.025)$. Hence, the average sero-prevalence over the five-year period according to age-groups was as follows: $16-20$ years $(10.2 \%), 21-25$ years (25.6\%), $26-30$ years ( $29.8 \%), 31-35$ years $(20.0 \%)$, and $\geq 36$ years (14.3\%) (Figure 1).

\section{Summary of our study findings}

Overall syphilis prevalence rates amongst registered pregnant women attending public antenatal clinics in Gaborone decreased from $2.96 \%$ in 2004 to $1.15 \%$ in 2008 . Trends in age specific syphilis prevalence per total number of reactive VDRL/RPR were highest amongst pregnant women aged $26-30$ years $(29.8 \%)$ and lowest amongst pregnant women aged $16-20$ years (10.2\%) for 2004-2008.

Table 1: Trends in syphilis sero-positivity amongst pregnant women attending public antenatal clinics in Gaborone, 2004-2008

\begin{tabular}{|l|}
\hline Year \\
\hline 2004 \\
\hline 2005 \\
\hline 2006 \\
\hline 2007 \\
\hline 2008 \\
\hline Total
\end{tabular}

$\begin{gathered}\text { N of pregnant women } \\ \text { registered for antenatal } \\ \text { care }\end{gathered}$
6418
6442
5681
5977
6703
31221

\begin{tabular}{|c|}
$\begin{array}{c}\boldsymbol{n} \text { of pregnant women } \\
\text { with reactive VDRL/RPR }\end{array}$ \\
\hline 190 \\
152 \\
99 \\
90 \\
77 \\
608
\end{tabular}

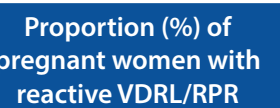

reactive VDRL/RPR

2.96
2.36
1.74
1.51
1.15
1.95

95\% Confidence interval (CI) $(p<0.001)$

$2.55-3.37$

$1.99-2.73$

$1.40-2.08$

$1.20-1.82$

$0.89-1.41$

$1.60-2.30$

Notes: VDRL = Venereal Disease Research Laboratory; RPR = Rapid Plasma Reagin. 


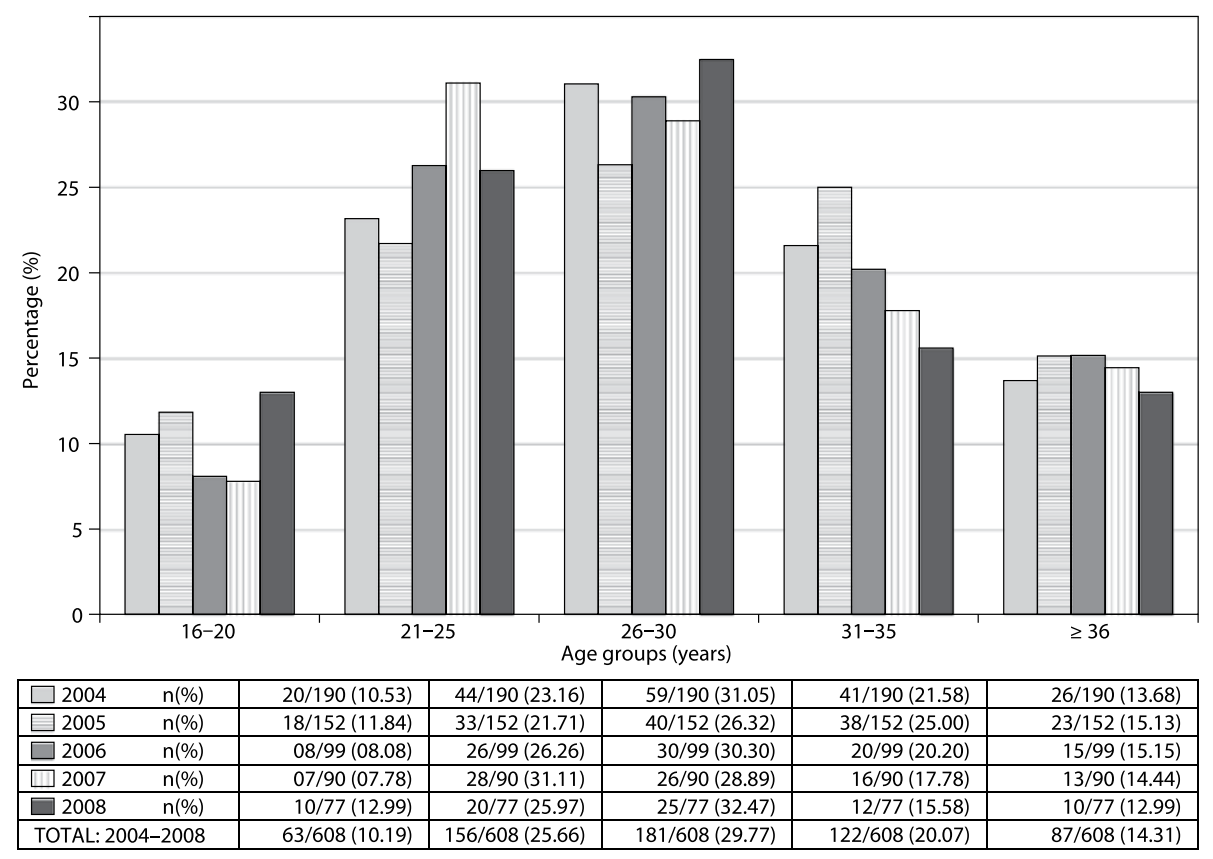

Figure 1: Trends in syphilis prevalence per total number of reactive VDRL/RPR by age group for combined clinics per year, 2004-2008.

\section{Discussion}

The results have shown that, at the time of the study, syphilis remained problematic in Gaborone, posing a risk for the poor outcome of pregnancy. The decline in the sero-prevalence could be explained by the introduction and implementation of the syndromic approach in the management of sexually transmitted infections for all sexually active age groups in the country more than a decade before the study. It is likely that this was further strengthened by population health education by the Botswana Ministry of Health which raised awareness on safe sex and easy access to health care services. ${ }^{10}$

The general trend in syphilis prevalence in the clinics studied showed a decline in syphilis prevalence rates consistent with that reported in a study conducted in Mozambique (14\% in 1998 to under $8 \%$ in 2004) $)^{14}$ and the South African National HIV and Syphilis survey (4.9\% in 2000 to $1.8 \%$ in 2006). ${ }^{9}$ The downward trend had already been observed in previous studies in Botswana ( $18 \%$ in 1993 to $2 \%$ in 2002$)^{10}$ and (4.8\% in 2002 to $3 \%$ in 2005$) .^{15}$ Rates of syphilis infection in our study were lower than those reported in the Francistown study ${ }^{16}$ and Botswana sentinel surveillance. ${ }^{17}$ This is probably due to the larger study sample used in this present study. Furthermore, most pregnant women in Gaborone are educated with a higher standard of living compared to women from Francistown, ${ }^{16}$ enabling them to make informed health choices and seek early health care.

Regarding trends in syphilis sero-prevalence by age groups over the five years, our study showed a steady increase in seroprevalence in the 21-25 year age-group, reaching its peak in the 26-30 year age-group, followed by a steady decline in the 3135 years and those 36 years and above. The reason for this upward trend followed by a downward trend in these age groups needs further investigation. However, it is noteworthy that on average, there has been a decline in sero-prevalence among each age group over the five-year period over which this study was conducted. This is probably as a result of the public health interventions implemented since the early nineties in Botswana to control the HIV epidemic, especially targeting the schoolgoing age-group. A further contributing factor was the introduction of the syndromic management of sexually transmitted infections at family planning clinics and free distribution of condoms, and also anonymous voluntary HIV counseling and testing. ${ }^{10}$ The low syphilis sero-positive among the 16 to 20 year age-group was comparable to a study conducted in South Africa among 15 to 19 year old pregnant women (1.9 to $2.1 \%),{ }^{9}$ and another study conducted in Tanzania from 2003 to 2004 among women aged 15 to 24 years (6.5\%). ${ }^{18}$

\section{Strengths and limitations of our study}

The main strength of this study aimed to examine trends in syphilis prevalence in clinics, which is an important and previously unexplored area in syphilis research in Botswana. The study population was large, enhancing the study statistical power (> 80\%) to allow generalisation of the study findings.

However, even though the researchers validated the data by comparing it with the data at the District Health Team central office which showed little difference, the study relied on recorded data with inherent data entry and processing errors.

\section{Implications for policy and practice}

This study could potentially serve as a scientific reflection on the current status of syphilis infection at antenatal care level in the country. It could inform national policies focusing on the control of syphilis among pregnant women and the population at large. Since syphilis as an infection is not confined only in the health sector, the study could also inform national programs involving other sectors like the social and economic sectors in combating the infection.

\section{Conclusions}

At the time the study was conducted, syphilis sero-prevalence among pregnant women in Gaborone showed decreasing levels from $2.96 \%$ in 2004 to $1.15 \%$ in 2008 . There was a high prevalence among pregnant women aged 26-30 year possibly due to lower use of condoms and late presentation for health care, while those aged 16-20 years showed the lowest incidence. There was also a marked variation in the trend of syphilis sero-prevalence 
by clinics. This necessitates further studies to investigate and establish the underlying associated factors.

Acknowledgement - This study was conducted in partial fulfillment of the requirements for the award of the Masters of Public Health (MPH) degree at the University of Liverpool, United Kingdom.

Competing interests - The authors declare that they have no financial or personal relationships which may have inappropriately influenced them in writing this article.

\section{References}

1. Holman KM, Wolff $M$, Seña $A C$, et al. Rapid plasma reagin titer variation in the 2 weeks after syphilis therapy. Sex Transm Infect. 2012;39(8):645-7.

2. Shah SA, Kristensen S, Memon MA, et al. Prevalence of syphilis among antenatal clinic attendees in Karachi: imperative to begin universal screening in Pakistan. J Pak Med Assoc. 2011;61(10):993-7.

3. Pahuja S, Gupta SK, Pujani $M$, et al. Treponema pallidum hemagglutination assay seroreactivity among healthy Indian donors and its association with other transfusion transmitted diseases. Asian J Transfus Sci. 2014;8(2):109-12.

4. World Health Organisation. Global prevalence and incidence of selected curable sexually transmitted infections. WHO. 2001. Available from: http://www.who.int/doctore/hiv/GRSTI/005.htm

5. World Health Organisation. Syphilis: symptoms, treatment and prevention. WHO. 1999. Available from: http://www.who.int/doctore/ hiv/GRSTI/005.htm

6. Genc M, Ledger WJ. Syphilis in pregnancy. J Sex Transm Dis. 2000;76:73-9.

7. Mullick S, Watson-Jones D, Beksinska M, Mabey D. Sexually transmitted infections in pregnancy: prevalence, impact on pregnancy outcomes, and approach to treatment in developing countries. Sex Transm Infect. 2005;81:294-302

8. Gloyd S, Chai S, Mercer MA. Antenatal syphilis in sub-Saharan Africa: missed opportunities for mortality reduction. Health Policy Plan. 2001;16:29-34.

9. Department of Health, 2009. 2008 National Antenatal Sentine HIV and Syphilis Prevalence Survey, South Africa. Available from: http://www.gov.za/sites/www.gov.za/files/HIV\%20SURVEY\%20 REPORT\%204\%200ct\%202008.pdf.

10. Creek TL, Thuku H, Kolou B, et al. Declining syphilis prevalence among pregnant women in northern Botswana: an encouraging sign for the HIV epidemic? Sex Transm Infect. 2005;81:453-455.

11. Romoren M, Rahman M. Syphilis screening in the antenatal care: a cross-sectional study from Botswana. BMC Int Health Hum Rights. 2006;6:8.

12. Botswana Ministry of Health-MOH. Botswana 2005 second generation HIV/AIDS surveillance. Gaborone: Government Printer; 2006.

13. Gaborone City Council-GCC. Annual antenatal statistics. District Health Statistics. Gaborone District, Botswana; 2006.

14. Lujn J, Arrazola de Onate W, Delva W, et al. Prevalence of sexually transmitted infections in women attending antenatal care in Tete province, Mozambique. S Afr Med J. 2008;98:46-51.

15. Gombachika BC, Fjeld H. In search of a face: Childbearing decisions among couples living with HIV from rural southern Malawi. Afr J AIDS Res. 2014;13(1):21-9.

16. Cenral Statistics Office - CSO. Population of towns, villages and associated localities. Population and housing census in Botswana. Gaborone: Government Printer; 2001.

17. Botwana Ministry of Health-MOH. Syphilis prevalence by districts Botswana Sentinel Surveillance 2007. Gaborone: Government Printer; 2007.

18. Swai RO, Somi GR, Matee MIN, et al. Surveillance of HIV and syphilis infections among antenatal clinic attendees in Tanzania-2003/2004. BMC Public Health. 2006;6:Article 91.

Received: 19-02-2015 Accepted: 02-11-2015 